\title{
Exhibition Session of the exp.at'11 International Conference
}

\author{
http://dx.doi.org/10.3991/ijoe.v8iS1.1931
}

Man has been the humanity's engineer for ages- human knowledge has been continuously built on conceptualization based on constant learning by doing activities. The observation and understanding of concepts and principles in action are the bases of the experimental activity leading to the correlation between reality and theory.

Experimental activities are really important, contributing to prepare the future engineers for solving practical problems and for consolidating theoretical understanding and volatile knowledge based on abstract concepts.

Therefore, experimental activity has been an essential part of education and training for engineers and engineering scientists.

exp.at'11 (the 1 ${ }^{\text {st }}$ Experiment@ International Conference) has been the first event of a new International Conference Experiment@ series devoted to online experimentation, enlarging the world capabilities in this particular area and contributing to collaborative work in emergent technologies, bringing together engineers, researchers and professionals from different areas. In its Exhibition Session, participants demonstrated their systems within developments of educational, medical and industrial applications.

In order to clarify all different terms to be used in this Special Issue it is important to summarize the related terminology. So, a remote laboratory is a set of experiments using real equipment in a physically "live" laboratory, enhanced by software applications and technologies, connected to the Internet and enabling remote access to real setups and their devices. A virtual lab is a set of experiments simulating experimental procedures and devices. They can be remotely accessed or downloaded to the user computer. A hybrid laboratory is a set of experiments using remote equipment and virtual simulations, providing both remote and virtual experiments in effective interaction. This means, it provides real experiments and software simulations in one single environment, [1]. An online Lab is a laboratory including all or some of those types of labs, [2].

Online experimentation is using emerging technology for allowing the access to those of the above labs of educational natures or to others of industrial, medical or research type, relying on online access.

Virtual reality involves software applications in which real environments are replicated with such a high level of realism that gets the user immersed as in real situations. In virtual reality senses as sight, sound and even touch can be explored.

At the education level, many reasons have been argued and discussed during the last decade concerning the use of those technologies in Engineering Education (EE), but the most important reason for the use of those tools in EE is simply the fact that technology is in our everyday life and young people will be more and more dependent on it in the future, either as simple human being or as expert engineers.

In addition they are also very important tools for research activities, industrial and medical training, or even for medical care since very early days, [3-5].

Virtual reality and haptics interaction is another promising area, mainly because of the increasing number of applications in industrial and medical fields largely used for training purposes.

At industrial level, virtual reality and haptics interaction are important tools for improving product design optimization by testing product assemblability, for planning assembly processes, for assembly training activities and so for improving assembly time and costs [6]. They are still explored in many other different ways, as task assistance in telemanipulation. Another important issue is e-manufacturing with the use of advanced and emerging information 
technologies to provide automated, data-driven productivity optimization. In this framework, expensive, specialized equipment is not always controlled in the same place or locale as the operator, and the development of remote lab technologies and the availability of collaborative virtual world environments enable to remotely control devices in order to design and build new products, train operators, and maintain systems [7].

At medical level a strong investment in training activity has increased the use of virtual reality tools, too. In surgery, for example, young doctors can achieve a sense of touch with a higher degree of realism when compared with surgery practice in cadavers. In addition, guided lessons are available for intensive training without clinician supervision [8].

At research level, experiments represent essential components of the research methodology in natural sciences and engineering, and the availability of virtual knowledge spaces has a crucial relevance in scientific cooperation and in the learning-by-doing process. The insufficient accessibility of experimental capacities affects mostly cost-intensive experimental equipment. In a particular way, this affects nanotechnology and robotics which have an extraordinarily influence on current technological developments [3]. In the learning-by-doing context, remote experimentation provides access to different types of systems, offering learning experiences ranging from experimentation to real-world problem solving, and taking advantage of the Internet and of a diversity of emerging communication, visualization, and simulation technologies. This represents the opportunity for researchers without immediate access to expensive specialized equipment or extremely rare scientific instruments, to conduct their research that would otherwise be beyond their reach and work [9].

This special issue offers nine contributions presented at the exhibition session of exp.at'11. A very brief outline of each demo is made in the next paragraphs.

Remote laboratories: bridging university to secondary schools presents the e-lab at Instituto Superior Técnico (IST) in Lisbon, Portugal, a framework used for offering remote labs in physics from post-graduate to secondary schools, where these resources can bring new infrastructure and new sources for learning and for dissemination of experimental activity. An authentication procedure is available. A novel attribute of e-labs, between others, is related with interesting and diversified experiments in physics, adapted for a broad target public.

Remote Laser Laboratory: First Demonstration hosted at Bauman Moscow State Technical University (BMSTU), Russia, demonstrates a laser engineering lab typifying complexion expensive equipment, dangerous for students' direct contact, one of the important reasons for making experimental resources remotely available. Laser set-ups always require very difficult tuning procedures, and so they need very special local handling for hands-on activity. Remote access overcomes this local interference and allows the systems to remain tuned for long term use.

Virtual Reality and Haptics for Product Assembly from Mechanical Engineering Institute-Polo FEUP (IDMEC-Polo FEUP), Porto, Portugal, reports on a software application with haptics interaction developed for demonstrating how efficient a virtual system is for assembly planning and training. A Phanton device from Sensable is used. Through this device all types of virtual touch as collision detection, geometry constraint recognition, gravity... are perceived. This increases the possibility of optimizing product design and assembly process and of training technicians in product assembly. All this features contribute for lowering product costs and increase productivity.

Exploring Images: A Haptic Mouse Approach, from Indiana University South Bend, USA, gives an idea about the use of inexpensive haptics mouse for "virtual touch" images. Examples based in the interaction with a Mona Lisa picture, a digital mammography and a labyrinth wall were demonstrated. By modulating the mouse parameters according to the image pixel information, the virtual information can be perceived at early stages of haptics use. This type of solution is very important for introducing the potentiality of this technological feature not so far from our everyday life, as is the case of a simple mobile in vibration mode... 
WebLab-Deusto-CPLD: A Practical Experience, from University of Deusto, Spain, provides a remote platform structure (presently, WebLab-Deusto 4.0M1) for Programmable Logic, offering diversity in experiments in the logic topic and it also includes demos, mobile lab, Facebook integration and other different options for students. This system is free of using any operating system through any web browser, and without specific security problems for communication ports and firewalls. A registration procedure is available.

Interactive Demonstration of a Remote Reconfigurable Logic Laboratory for Basic Digital Design, from University of Coimbra, Portugal, offers flexible additional working hours to freshman students in the topic of the Field Programmable Gate Array. It uses an AlteraDE2 FPGA board and allows online programming, online testing and remote observation through a web cam, overcoming the already traditional simulators. A registration procedure is available.

Virtual Reality applied to dental implant surgery - a computer guided protocol -, used at Pinheiro Torres Dental Clinic in Porto, Portugal, shows the highly technical procedures available for dental implants, which guide the dental clinician to follow accurately a protocol procedure. This virtual programming procedure permits to explore the best structural conditions for the surgery, reducing the number of laboratory and surgical sessions, restricting the invasive surgery to one-step process and the overall treatment time, improving the post-operative symptoms and reducing overall risks.

Haptic Positioning Environment for the Hip Resurfacing Surgery, from Mechanical Engineering Institute (IDMEC-Polo IST), Lisbon, Portugal, presents a procedure under development looking to improve novice surgeon training in the "insertion of the hip resurfacing surgery guide wire” with high accuracy and improved safety. For that end, a virtual model reproducing the human environment, a model for simulating interaction during driller-bone process and a force field for implementing virtual touch are needed. An attractive feature is the use of a very low cost haptics device with 3 DOF, from Falcon series.

Physics Experiments at the UNEDLabs Portal - A network of remote and virtual laboratories for physics education, from Distance National University, Madrid, Spain, offers a framework, FisL@bs, based in the open source LMS Moodle. Remote and virtual labs are available and an interesting set of experiments in the physics area is available. They are also of interest for many engineering fields where fundamental concepts are needed, as is the case of experiments in elasticity, electricity and electromagnetism, optics,.... The Moodle potentialities are extensively explored. An automatic booking system has been developed by making a compatible interface between the Moodle and the system's hardware.

All these nine demos have been available for all the exp.at'11 participants, for discussions and for sharing of experiences for 2 hours, bringing to the conference a high level of participation and enthusiasm, which we thank to all.

The Guest Editors wish to thank the Experiment@ International Conference 2011 main sponsor, Calouste Gulbenkian Foundation, Portugal.

A word of gratitude is also due to the University of Porto and to its Faculty of Engineering, to the University of Coimbra and to its Faculty of Sciences and Technology, to Ordem dos Engenheiros da Região Centro and to the Associated Laboratory of Aeronautics, Energy and Transports, all Portuguese Institutions. We also thank to all the Collaborating Institutions: IAOE and IGIP in Austria and SPEE, MTBrandão, Santander Totta, Unicer and Fundação para a Computação Científica Nacional in Portugal.

The last thanks go to the Editor-in-Chief of the International Journal of Online Engineering (iJOE), Prof. Michael E. Auer.

The Guest editors,

Maria Teresa Restivo, Faculty of Engineering of University of Porto and LAETA

Alberto Cardoso, Faculty of Sciences and Technology of University of Coimbra 


\section{References}

[1] D. G. Zutin, M. E. Auer, C. Maier, M. Niederstätter, Lab2go - A Repository to Locate Educational Online Laboratories, (2010), pp 1741-1746, IEEE EDUCON 2010, Madrid, Spain.

[2] http://www.lab2go.net/lab2go/metadata, accessed on 13/01/2012.

[3] Jeschke, S. ; Gramatke, A.; Pfeiffer, O. et al. "Networked virtual and remote laboratories for research collaboration in natural sciences and engineering", Proceedings of the 12th IASTED International Conference on Computers and Advanced Technology in Education - CATE 2009.

[4] Pradarelli, B.; Latorre, L.; Flottes, M.-L. et al. "Remote Labs for Industrial IC Testing", IEEE Transactions on Learning Technologies, 2(4): 304-311, 2009. http://dx.doi.org/10.1109/TLT.2009.46

[5] F.K. HERBIG, J.L. DILY, N.E. (GOOCH, R.M. DONATI AND J.W. FLETCHER Diagnostic Services and communication protocols for remote nuclear medicine laboratories, ProcAnnuSympComputAppl Med Care. 1980 November 5; 1: 136-141.

[6] Xia, P., Lopes, A.; Restivo, M. T. et al. "A New Type Haptic-based Virtual Environment System for Assembly Training of Complex Products". International Journal of Advanced Manufacturing Technology, 2011. http://dx.doi.org/10.1007/s00170-011-3381-8

[7] J. Fayolle, C. Gravier, N. Yankelovich and E. Kim (2011), Remote Lab in Virtual World for Remote Control of IndustrialProcesses. Proceedings of the 2011 IEEE International Conference on Multimedia and Expo (ICME). http://dx.doi.org/10.1109/ICME.2011.6012049

[8] Kimin Kim, Jinah Park (2009), Virtual Bone Drilling for Dental Implant Surgery Training. VRST 2009, Kyoto, Japan, November 18-20

[9] Marilyn M. Lombardi (2007), Authentic Learning for the 21st Century: An Overview. Educause Learning Initiative - advancing learning through IT innovation, http://www.educause.edu/ELI/AuthenticLearningforthe21stCen/ 156769 , accessed on 13/01/2012.

[10] Horácio Fernandes, "Remote Laboratories: Bridging University to Secondary Schools", Int. J. of Online Engineering (iJOE), Vol. 8, S1, January 2012, pp. 8-9, http://dx.doi.org/10.3991/ijoe.v8iS1.1902

[11] Igor Titov, "Remote Laser Laboratory: First Demonstration", Int. J. of Online Engineering (iJOE), Vol. 8, S1, January 2012, pp. 10-11, http://dx.doi.org/10.3991/ijoe.v8iS1.1891

[12] Pingjun Xia, Antonio M Lopes, Maria Teresa Restivo, "Virtual Reality and Haptics for Product Assembly", Int. J. of Online Engineering (iJOE), Vol. 8, S1, January 2012, pp. 12-14, http://dx.doi.org/10.3991/ijoe.v8iS1.1894

[13] James Wolfer, "Exploring Images: A Haptic Mouse Approach", Int. J. of Online Engineering (iJOE), Vol. 8, S1, January 2012, pp. 15-16, http://dx.doi.org/10.3991/ijoe.v8iS1.1889

[14] Javier Garcia-Zubia, Ignacio Angulo, Pablo Orduna, Unai Hernandez, Diego Lopez-de-Ipina, Luis Rodriguez, Olga Dziabenko, Veronica Canivell, "WebLab-Deusto-CPLD: A Practical Experience", Int. J. of Online Engineering (iJOE), Vol. 8, S1, January 2012, pp. 17-18, http://dx.doi.org/10.3991/ijoe.v8iS1.1952

[15] Jorge Lobo, "Interactive Demonstration of a Remote Reconfigurable Logic Laboratory for Basic Digital Design", Int. J. of Online Engineering (iJOE), Vol. 8, S1, January 2012, pp. 19-20, http://dx.doi.org/10.3991/ijoe.v8iS1.1886

[16] J. Pinheiro Torres, "Virtual Reality Applied to Dental Implant Surgery - A Computer Guided Protocol", Int. J. of Online Engineering (iJOE), Vol. 8, S1, January 2012, pp. 21-22, http://dx.doi.org/10.3991/ijoe.v8iS1.1956

[17] Pedro Santos Pires, Pedro Teodoro, Jorge Martins, José Sá da Costa, "Haptic Positioning Environment for the Hip Resurfacing Surgery", Int. J. of Online Engineering (iJOE), Vol. 8, S1, January 2012, pp. 23-25, http://dx.doi.org/10.3991/ijoe.v8iS1.1890

[18] Sebastian Dormido, José Sánchez, Luis de la Torre, Ruben Heradio, Carmen Carreras, Juan pedro Sánchez, Manuel Yuste, "Physics Experiments at the UNEDLabs Portal", Int. J. of Online Engineering (iJOE), Vol. 8, S1, January 2012, pp. 26-27, http://dx.doi.org/10.3991/ijoe.v8iS1.1945 